\title{
Research on Quality Tests for Shop Floor Application Based on Local Network
}

\author{
TanLi Mengqing \\ School of Mech. \& Eng. \\ University of South China \\ Hunan, Hengyang, China, 421001 \\ TLMQ-TEAM@163.com
}

\author{
Jiang Yan, Wang Xiang, Wang Yulin, Peng Rushu \\ School of Mech. \& Eng. \\ University of South China \\ Hunan, Hengyang, China, 421001 \\ TLMQ-TEAM@163.com
}

\begin{abstract}
In order to improve the efficiency of quality tests and optimize the process of the quality control, this paper deeply investigates main aspects of quality tests for shop floor application. The hierarchical model of quality tests is firstly set up in this paper. After the layout of hardware and network is analyzed, the integration of software for shop floor application is introduced. Advanced technology is guide post all the time, and some advanced technology applied in quality tests is discussed in this paper, including apparatus and instrument technology, data acquisition and communication link technology, and computer programming technology.
\end{abstract}

Keywords—shop floor application, quality testing, local network, advanced thechnology

\section{INTRODUCTION}

The trend of quality testing for shop floor is generalization, automation, integration and digitalization. Generalization mainly embodies that the tester and device are more and more generalized and standardized including its function and communication interface. And automation includes control automation and management automation, the former mainly implies that the tester and testing line have automatic function and the later mainly embodies that the data processing of quality management is more and more done by computer. Integration is a focused point [1], where the integration among computers used in quality testing become a mature technology besides the integration of tester and device, and Intranet concepts generated with the development of new communication technology, which is the development of Internet technology in enterprise applications, and enterprise Intranet breaks the information isolation of shop floor, realized an information integration of the device, group, and workshop. Digitalization is an update and new achievement for quality test because of the easiness of cooperation with computer processing, which decreases the manual error and tiredness of manual labor [2, 3].

Shop floor is the base of quality testing for manufacturing enterprises, not only testing equipment and devices locate in the shop floor, but also the most of quality testing clerk are subjected to shop floor, and the main point of quality affair is done in the shop floor. At the same time, local network with advanced router become an important implementing type of integration for shop floor application. [4]

\section{The HIERARCHY MODEL OF QUALITY TeStS}

The quality test for shop floor application based on local network can be divided into three layers: device layer, data disposal layer, and monitoring layer and the detail is shown in figure 1 . The device layer and data disposal layer usually refer to inspection station team of workshop, and monitoring layer is generally responsible to quality control division of factory [5].

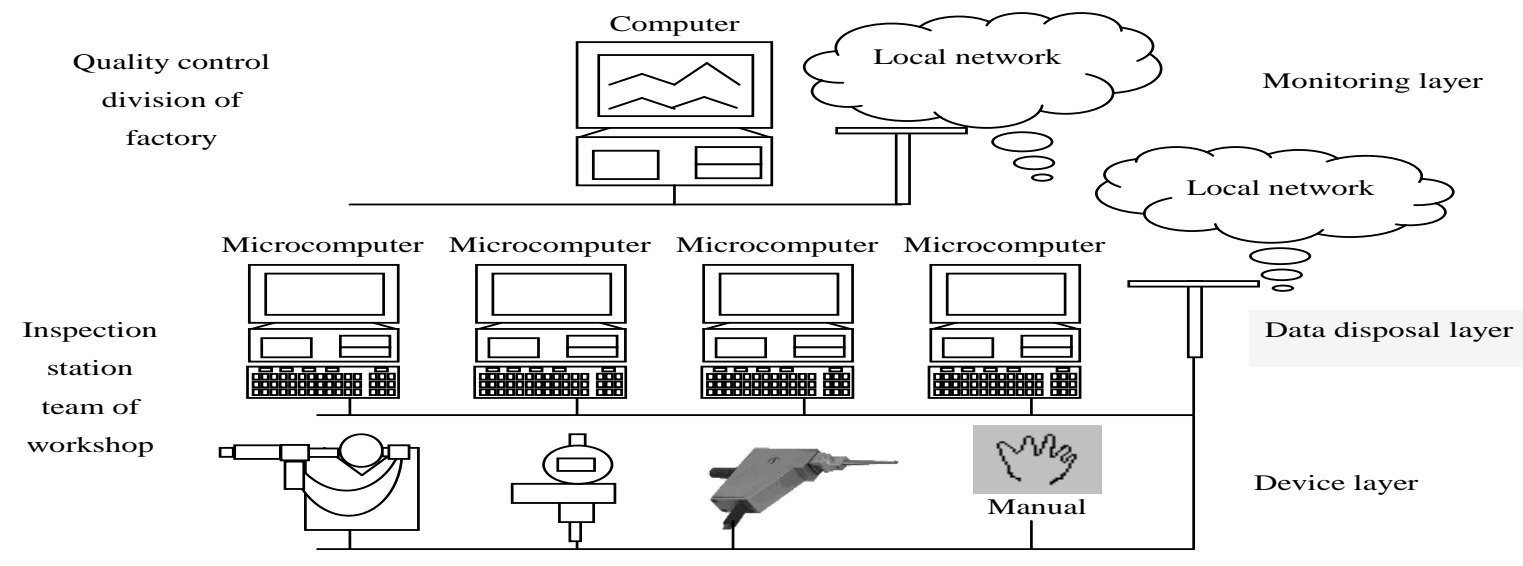

Fig. 1. Hierarchical model of quality test 
The device layer mainly includes testing instrument and detection device, which is the constitutive base of quality test. The second layer, data disposal layer is the work foundation of quality test, which is responsible to the parts sampling and inspection, to judge if it is qualified or not, then eliminate the scrap, and achieve the quality data collection, in this layer it have to guarantee that the quality data is accurate and reliable. The monitoring layer is the third layer of quality test, which is responsible to check, analyze, control and correct the quality information submitted by the lower layers.

\section{NETWORK STRUCTURE OF QUALITY TESTS FOR SHOP FLOOR APPLICATION}

Network of quality test for shop floor application aims to integrate the equipment and hardware of quality testing. In this local network, a microcomputer acts as quality testing station, by which, quality testing is divided into two parts: process test and finish test. And gateway note computer is used as an upper computer in monitoring layer. The implementation of experimental network of quality testing is carried on with the technology support of universal communication interface and local network with general router. By actual running of the network, the result proved that the network structure as figure 2 is reasonable, and the technical program is executable $[6,7]$.
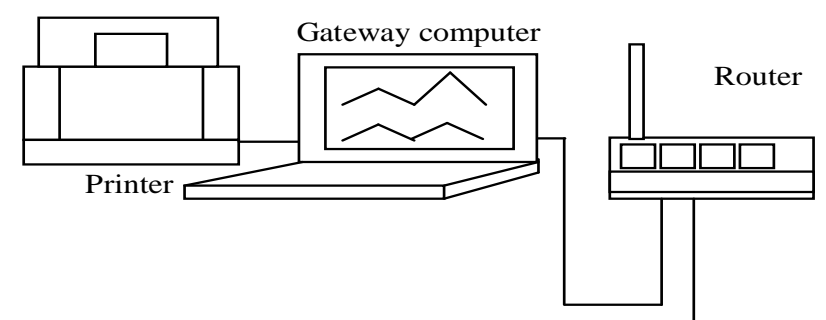

Microcomputer

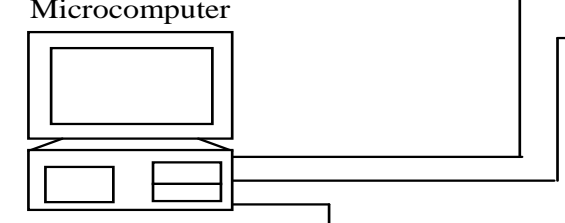

Quality test station

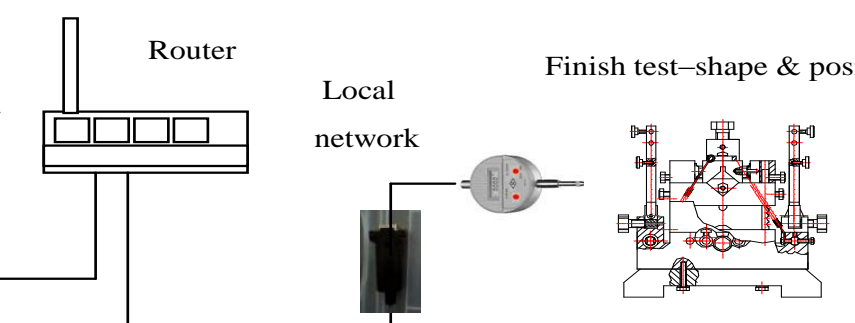

Finish test-dimension

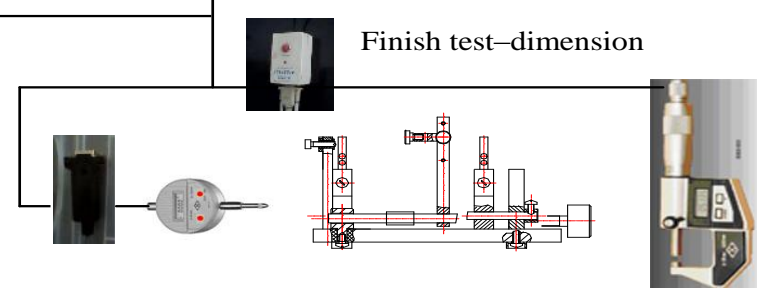

Communication link

Process test-offline

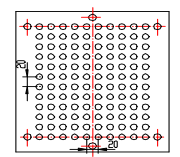

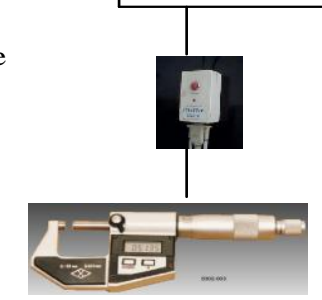
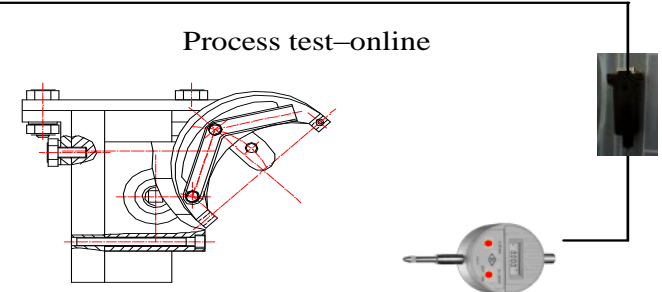

Fig. 2. Network structure of quality test for shop floor application

\section{SOFTWARE INTEGRATION OF QUALITY TESTS FOR SHOP FLOOR APPLICATION}

In the quality test for shop floor application, there is a number of software for data acquisition and to process the quality data [8]. There are some application program are developed by tester manufacturing factory and some application of Microsoft Windows system are also used for quality data processing, all these application program are integrated into our quality test program for shop floor application. The detail is shown in figure 3 . 


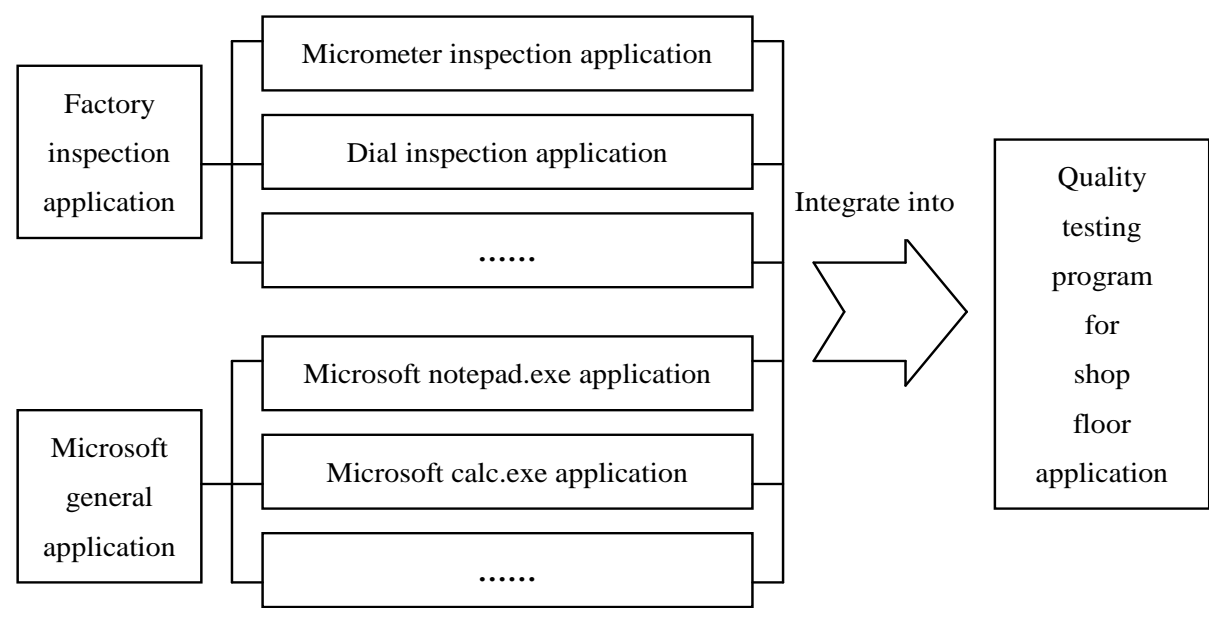

Fig. 3. Software integration of quality test for shop floor application

\section{APPLICATION OF ADVANCED TECHNOLOGY IN QUALITY TESTS}

In quality testing, all kinds of advanced technologies are to be gradually adapted, such as automatic detection, integration, and digital measurement and so on.

But, if the condition of the factory is unfavorable, the usable and effective technique and device should be remained. For example, display values of various measuring instruments can be read out in manual, and take it as the results of machining precision detection of rotational shape part; on the other hand, for factory with good conditions, instrument interfaces and acquisition part for testing can be applied to improve efficiency, and computers can be linked with router. And record inspection results are saved in data storing media, then printing is used as saving and report, it also can achieve data communications with upper management layer.

\section{A. Apparatus and Instrument Technology}

Apparatus and instrument is the base and support for quality testing. To develop in-process and on-line inspection technology is a main trend for advanced testing technology, the figure 4 is the apparatus of on-line inspecting for cutting. In the condition of shop floor, apparatus and instrument should meet some special requirements:

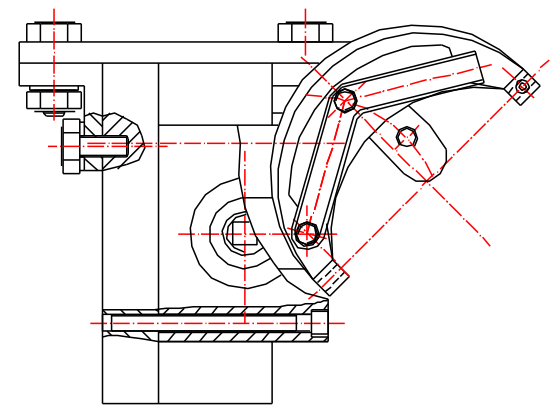

Fig. 4. apparatus of on-line testing for cutting
- Temperature correction Generally, because the temperature of shop floor is changing and not consisted with the standard measuring condition, the measuring result should be corrected.

- Anti-abrasion technology for contact measuring, (a) Anti-abrasion of moving part:For machinery, moving part is generally a very important section. Tiny displacement is needed in tester, so that anti-abrasion of tiny moving part is a main aspect for careful consideration. For example, in order to consider the abrasion of moving guide rail, the sleeve is added for the base of guide rail in apparatus. (b) Anti-abrasion of alterable probe:In the tester, probe is the most serious section for emerging abrasion. There are two measures to solve, one is altering, and another is mending. The former is more convenient and more successive.

- Instrument error calibration, In the procedure of using, the emerging of error for apparatus and whole instrument is unavoidable. The technology of selfmaintaining and self-calibrating will become a new mode for instrument error calibration.

\section{B. Data Acquisition and Communication Link Technology}

\section{1) Communication interface of tester and device}

Testers and devices in quality testing for shop floor need to data exchanging each other, their data communication usually have two types: (1) RS232C, and USB. RS232C is an older method for computer interface, and USB is now widely used in external equipment of the computer. The foot layout of RS232C and profile of USB are shown in figure 6.

\section{2) Communication router of testing computer}

In quality testing for shop floor, there are usually a few of computers for data acquisition and quality data processing. Router and local exchanger are two ways for communication among these computers, especially to construct local network of quality testing and information. The illustration of router for communication among these computers is shown in figure 7. 

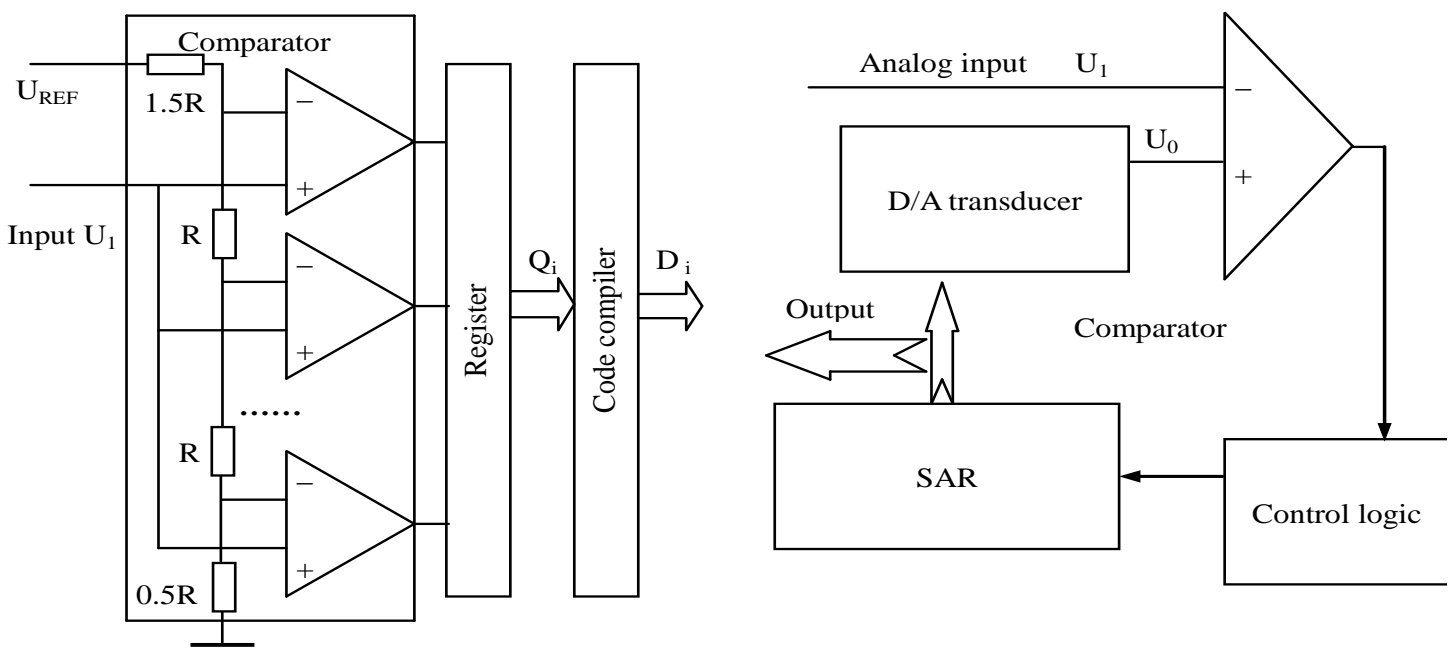

Fig. 5. Digital AD technology

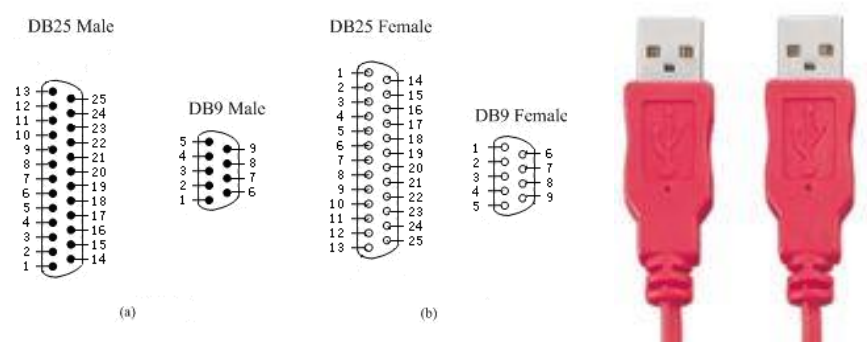

Fig. 6. Communication interface of tester and device

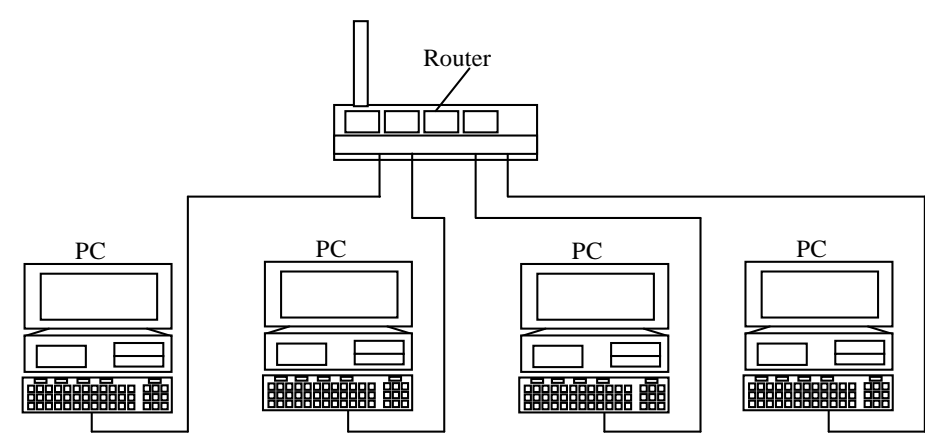

Fig. 7. Communication router of testing computer

\section{Computer Programming Technology}

Not only design of the program of data acquisition but also design of the processing application of quality data, the computer programming technology is very important. In the quality testing, many application programming are developed by object-oriented model and universal modeling language, and main programming tool is $\mathrm{VC}++$. Using the object- oriented programming language, the class and instance are the core concept, and the usual class diagram in the programming of quality testing is shown in figure 8 . 


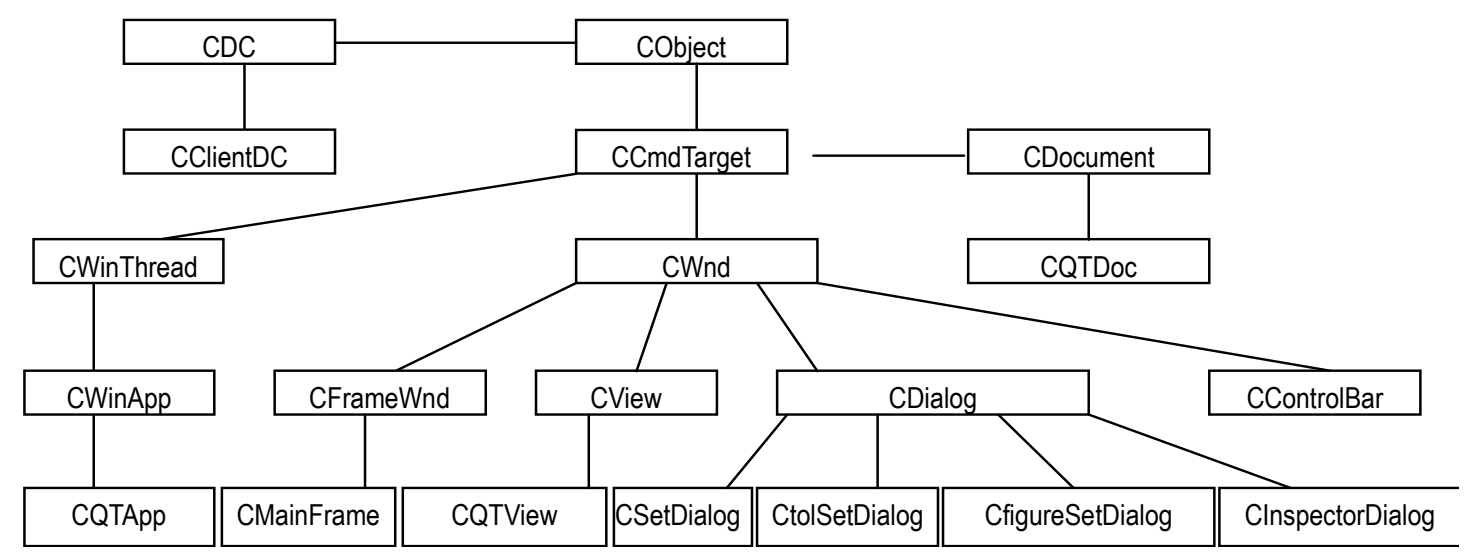

Fig. 8. Class diagram of application program

It is different for programming with update tool and language, as $\mathrm{VC}++$, than the past method based on process. Besides the inheritable property, the derivation is also the main feature for object-oriented programming. In the quality testing programming, many derivation classes are inherited from CDialog, CControlBar, etc. At the same time, the implementation of class usually is carried out by some member function, for the quality testing, using computer programming to implement the drawing of data curve is a very important application, the figure 9 descript the timing order of drawing which is applied in most application of the quality testing program.

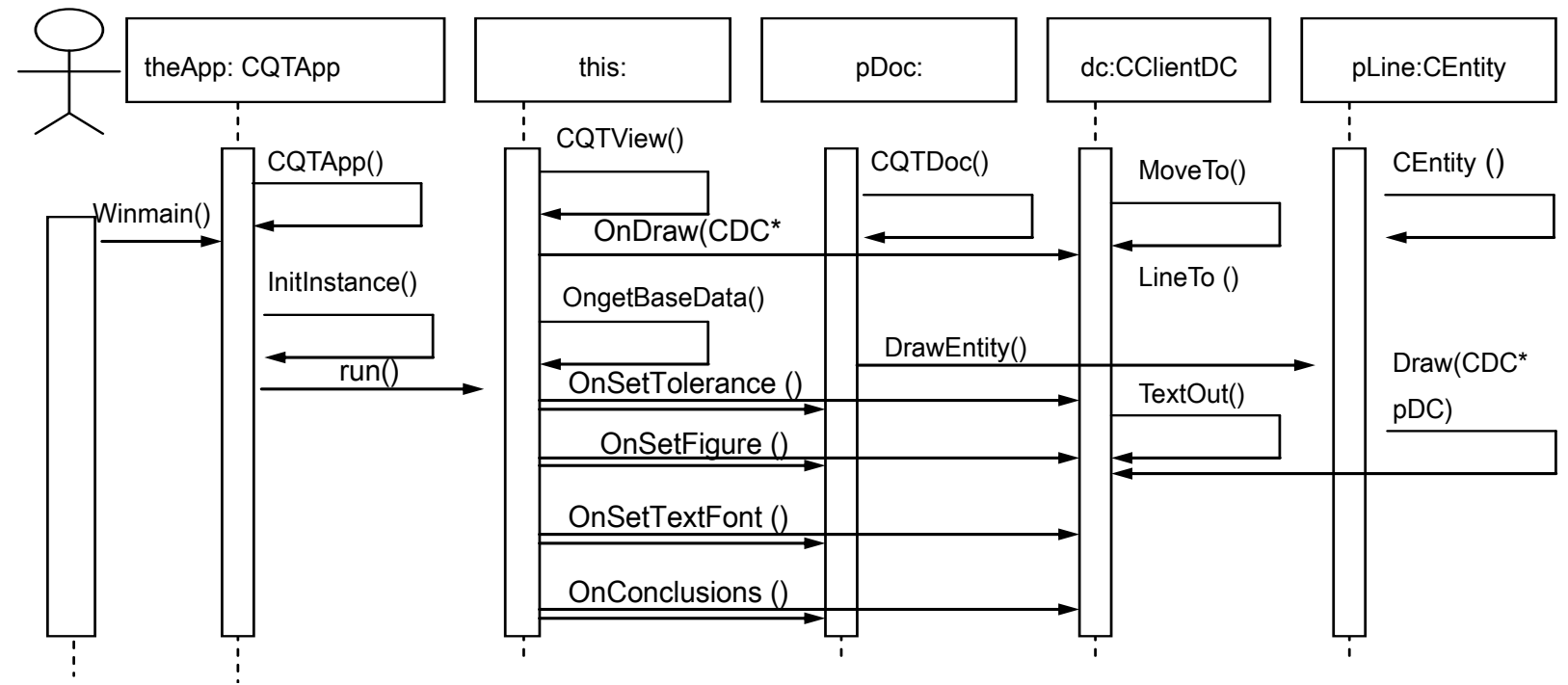

Fig. 9. Timing diagram of drawing of quality testing curve

\section{CONCLUSIONS}

Shop floor is the base of manufacturing factory, and the application of shop floor is also the base for the quality testing. With the development and using of mature advanced technology, the quality test for shop floor application based on local network will become the focus and get great achievement. The quality test for shop floor application based on local network can be generally divided into three layers: device layer, data disposal layer, and monitoring layer. The implementation of experimental network of quality testing based on local network is carried on with the technology support of universal communication interface of tester and with general router in the communication among computers. Application of advanced technology in quality test should include three aspects: (1) Apparatus and instrument technology, (2) Data acquisition and communication link technology, (3) Computer programming technology.

\section{REFERENCES}

[1] GENG Hong qin, ZHANG Yuanying, Wireless Network and the Method of Building, Journal of Tianzhong, 2002, 5, pp. 24-25(In Chinese).

[2] Zhang Gongxu, New quality management, High education press, May, 1998(In Chinese).

[3] Wu Liaoyuan, Research on intelligent quality \& diagnosis technologies and the application in CIMS, April, 2007(In Chinese).

[4] LIN Zhihang. The computer aided quality system [M]. Beijing, Mechanical Industry Press, 1996(In Chinese).

[5] TanLi Mengqing, Zhang Qian, Peng Rushu,et al., On Hierarchical Quality Monitoring System Based on Wireless Intranet, 211 
International Conference on Information and Industrial Electronics(ICIIE 2011), January 14-15,2011, Chengdu, China.

[6] TanLi Mengqing, Development and simulated test of WEBSPC, IDDME \&ACCSPI2008, Beijing, Sept., 2008.

[7] Zhao Haibin, Nabil Anwer, Pierre Bourdet, Wang Junying, Multisensor Integration for Coordinate Metrology, IDDME \&ACCSPI2008, Beijing, Sept., 2008.

[8] A.Kusiak, T.Letsche and A.Zakarian. "Data modeling with IDEF1x, International Journal of Computer Integrated Manufacturing", 1997, 10 (6): pp.470-487. 